\title{
Multi-criteria selection of manufacturing processes in the conceptual process planning
}

\author{
Lukic, D. ${ }^{a,}{ }^{,}$, Milosevic, M. ${ }^{a}$, Antic, A. ${ }^{a}$, Borojevic, S. ${ }^{b}$, Ficko, M. \\ ${ }^{a}$ University of Novi Sad, Faculty of Technical Sciences, Novi Sad, Serbia \\ bUniversity of Banja Luka, Faculty of Mechanical Engineering, Banja Luka, Bosnia and Herzegovina \\ 'University of Maribor, Faculty of Mechanical Engineering, Maribor, Slovenia
}

\begin{abstract}
A B S T R A C T
Process planning is one of the most difficult tasks in product development caused by the large number of technical, technological, economic, environmental and other criteria. Accordingly, the selection of manufacturing processes is a complex multi-criteria decision making problem since it considers a number of possible alternative manufacturing processes in addition to a large number of specified criteria. This paper represents the computer-aided methodology for the multi-criteria evaluation and selection of manufacturing processes at the stage of conceptual process planning. The developed methodology is primarily focused on the mapping of product design and manufacturing requirements. Manufacturing processes that fail to meet the given conditions on the basis of 10 criteria such as materials, production volume, productivity, dimensional accuracy, surface finish, etc., are eliminated according to the developed rules. Then, the multi-criteria evaluation and ranking of manufacturing processes is performed based on 5 criteria: manufacturing cycle time, process flexibility, material utilization, quality and operating costs. Based on this methodology, a system is developed for the multi-criteria selection of manufacturing processes, whose implementation is presented in the case of the hip joint endoprosthesis.
\end{abstract}

\section{ARTICLE INFO}

Keywords:

Manufacturing processes

Conceptual process planning

Multi-criteria decision making

Process selection

*Corresponding author:

lukicd@uns.ac.rs

(Lukic, D.)

Article history:

Received 26 January 2017

Revised 24 February 2017

Accepted 26 May 2017

\section{References}

[1] Unger, D., Eppinger, S. (2011). Improving product development process design: A method for managing information flows, risks, and iterations, Journal of Engineering Design, Vol. 22, No. 10, 689-699, doi: 10.1080/ 09544828.2010 .524886$.

[2] Chang, K.-H. (2013). Product cost estimating, In: Product manufacturing and cost estimating using CAD/CAE, Academic Press, Boston, 237-294, doi: 10.1016/B978-0-12-401745-0.00006-X.

[3] Dieter, G.E . (1997). ASM Handbook: Volume 20: Material selection and design, ASM Intenational, Ohio, USA.

[4] Ashby, M.F. (2005). Materials selection in mechanical design, $3^{\text {rd }}$ edition, Elsevier Butterworth-Heinemann, Oxford, UK.

[5] Creese, R.C. (1999). Introduction to manufacturing processes and materials, Marcel Dekker Inc., New York, USA.

[6] Boothroyd, G., Dewhurst, P., Knigh, W.A. (2010). Product design for manufacture and assembly, $3^{\text {rd }}$ edition, Taylor \& Francis, New York, USA.

[7] Swift, K.G., Booker, J.D. (2003). Process selection: From design to manufacture, $2^{\text {nd }}$ edition, Elsevier ButterworthHeinemann, Oxford, UK.

[8] Xu, X., Wang, L., Newman, S.T. (2011). Computer-aided process planning - A critical review of recent developments and future trends, International Journal of Computer Integrated Manufacturing, Vol. 24, No. 1, 1-31, doi: $\underline{10.1080 / 0951192 X .2010 .518632 . ~}$

[9] Wang, J.-S., Liu, D., Duan, G., Lei, N. (1999). Analytic hierarchy process based decision modelling in CAPP development tools, The International Journal of Advanced Manufacturing Technology, Vol. 15, No. 1, 26-31, doi: 10.1007/ 
$\underline{\mathrm{s} 001700050035}$.

[10] Feng, S.C., Song, E.Y. (2003). A manufacturing process information model for design and process planning integration, Journal of Manufacturing Systems, Vol. 22, No. 1, 1-15, doi: doi: 10.1016/S0278-6125(03)90001-X.

[11] Chan, K., King, C., Wright, P. (1998). COMPASS: Computer oriented materials, processes, and apparatus selection system, Journal of Manufacturing Systems, Vol. 17, No. 4, 275-286, doi: doi: 10.1016/S0278-6125(98)80075-7.

[12] Xiao, W., Zheng, L., Huan, J., Lei, P. (2015). A complete CAD/CAM/CNC solution for STEP-compliant manufacturing. Robotics and Computer-Integrated Manufacturing, Vol. 31, 1-10, doi: 10.1016/j.rcim.2014.06.003.

[13] Febransyah, A. (2001). A feature-based approach to automating high-level process planning, PhD thesis, Faculty of

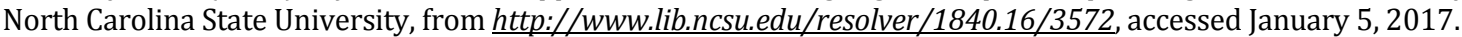

[14] Cay, F., Chassapis, C. (1997). An IT view on perspectives of computer aided process planning research, Computers in Industry, Vol. 34, No. 3, 307-337, doi: 10.1016/S0166-3615(97)00070-5.

[15] Gupta, S.K., Regli, W.C., Das, D., Nau, D.S. (1997). Automated manufacturability analysis: A survey, Research in Engineering Design, Vol. 9, No. 3, 168-190, doi: 10.1007/bf01596601.

[16] Dargie, P.P., Parmeshwar, K., Wilson, W.R.D. (1982). MAPS-1: Computer-aided design system for preliminary material and manufacturing process selection, Journal of Mechanical Design, Vol. 104, No. 1, 126-136, doi: 10.1115/ $\underline{1.3256302 .}$

[17] Shea, C., Reynolds, C., Dewhurst, P. (1989). Computer-aided material and process selection, In: Proceedings of the $4^{\text {th }}$ International Conference on Product Design for Manufacture and Assembly, Newport, USA, 399-414.

[18] Farris, J., Knight, W.A. (1992). Design for manufacture: expert processing sequence selection for early product design, CIRP Annals-Manufacturing Technology, Vol. 41, No. 1, 481-484, doi: 10.1016/S0007-8506(07)61249-9.

[19] Yu, J.-C., Krizan, S., Ishii, K. (1993). Computer-aided design for manufacturing process selection, Journal of Intelligent Manufacturing, Vol. 4, No. 3, 199-208, doi: 10.1007/bf00123964.

[20] Esawi, A.M.K., Ashby, M.F. (2003). Cost estimates to guide pre-selection of processes, Materials \& Design, Vol. 24, No. 8, 605-616, doi: 10.1016/S0261-3069(03)00136-5.

[21] Giachetti, R.E. (1998). A decision support system for material and manufacturing process selection, Journal of Intelligent Manufacturing, Vol. 9, No. 3, 265-276, doi: 10.1023/a:1008866732609.

[22] Smith, C., Wright, P., Séquin, C. (2003). The manufacturing advisory service: Web-based process and material selection, International Journal of Computer Integrated Manufacturing, Vol. 16, No. 6, 373-381, doi: 10.1080/ 0951192031000078176

[23] H'mida, F., Martin, P., Vernadat, F. (2006). Cost estimation in mechanical production: The cost entity approach applied to integrated product engineering, International Journal of Production Economics, Vol. 103, No. 1, 17-35, doi: 10.1016/i.ijpe.2005.02.016.

[24] Saric, T., Simunovic, G., Simunovic, K., Svalina, I. (2016). Estimation of machining time for CNC manufacturing using neural computing, International Journal of Simulation Modelling, Vol. 15, No. 4, 663-675, doi: 10.2507/IJSIMM15 (4)7.359.

[25] Denkena, B., Shpitalni, M., Kowalski, P., Molcho, G., Zipori, Y. (2007). Knowledge management in process planning, CIRP Annals-Manufacturing Technology, Vol. 56, No. 1, 175-180, doi: 10.1016/i.cirp.2007.05.042.

[26] Wang, J.F., Kang, W.L., Zhao, J.L., Chu, K.Y. (2016). A simulation approach to the process planning problem using a modified particle swarm optimization, Advances in Production Engineering \& Management, Vol. 11, No. 2, 77-92, doi: 10.14743/apem2016.2.211.

[27] Marri, H.B., Gunasekaran, A., Kobu, B. (2003). Implementation of computer-integrated manufacturing in small and medium enterprises, Industrial and Commercial Training, Vol. 35, No. 4, 151-157, doi: 10.1108/001978503 10479132

[28] Nguyen, V.D., Martin, P. (2015). Product design-process selection-process planning integration based on modeling and simulation, The International Journal of Advanced Manufacturing Technology, Vol. 77, No. 1, 187-201, doi: 10.1007/s00170-014-6446-7.

[29] Boral, S., Chakraborty, S. (2016). A case-based reasoning approach for non-traditional machining processes selection, Advances in Production Engineering \& Management, Vol. 11, No. 4, 311-323, doi: 10.14743/apem2016. $\underline{4.229}$.

[30] Klancnik, S., Brezocnik, M., Balic, J. (2016). Intelligent CAD/CAM system for programming of CNC machine tools, International Journal of Simulation Modelling, Vol 15, No. 1, 109-120, doi: 10.2507/IJSIMM15(1)9.330.

[31] Hodgett, R.E. (2016). Comparison of multi-criteria decision-making methods for equipment selection, The International Journal of Advanced Manufacturing Technology, Vol. 85, No. 5, 1145-1157, doi: 10.1007/s00170-015-7993-2.

[32] Singaravel, B., Selvaraj, T. (2015). Optimization of machining parameters in turning operation using combined TOPSIS and AHP method, Tehnički vjesnik - Technical Gazette, Vol. 22, No. 6, 1475-1480, doi: 10.17559/TV20140530140610.

[33] Park, H.-S., Nguyen T.-T., Kim, J.-C. (2016). An energy efficient turning process for hardened material with multicriteria optimization, Transactions of FAMENA, Vol. 40, No. 1, 1-14.

[34] Lukic, D. (2012). Development of a general technological preparation of production model, PhD thesis, Faculty of Technical Sciences, Novi Sad, Serbia, (In Serbian).

[35] Eniko, P., Sokovic, M., Kramar, D. (2016). Influence of non-productive operations on product quality, Strojniški vestnik - Journal of Mechanical Engineering, Vol. 62, No. 3, 197-204, doi: 10.5545/sv-jme.2015.3109.

[36] Simunovic, G., Svalina, I., Simunovic, K., Saric, T., Havrlisan, S., Vukelic, D. (2016). Surface roughness assessing based on digital image features, Advances in Production Engineering \& Management, Vol. 11, No. 2, 93-104, doi: 10.14743/apem2016.2.212.

[37] Čus, F., Župerl, U. (2015). Surface roughness control simulation of turning processes, Strojniški vestnik - Journal of 
Mechanical Engineering, Vol. 61, No. 4, 245-253, doi: 10.5545/sv-jme.2014.2345.

[38] Tabakovic, S., Zeljkovic, M., Zivkovic, A. (2014). General parametric model of the body of the total hip endoprosthesis, Acta Polytechnica Hungarica, Vol. 11, No. 11, 227-246, doi: 10.12700/APH.11.01.2014.01.14.

[39] Milosevic, M., Lukic, D., Antic, A., Lalic, B., Ficko, M., Simunovic, G. (2017). e-CAPP: A distributed collaborative system for internet-based process planning, Journal of Manufacturing Systems, Vol. 42, 210-223, doi: 10.1016/i.jmsy.2016. $\underline{12.010 .}$ 
APEM
Advances in Production Engineering \& Management

Letnik 12 | Številka 2 | Junij 2017 | Strani 151-162

https://doi.org/10.14743/apem2017.2.247
ISSN 1854-6250

Spletna stran: apem-journal.org Izvirni znanstveni članek

\title{
Večkriterijska izbira izdelovalnega postopka v fazi konceptualnega planiranja
}

\author{
Lukic, D. ${ }^{\text {a, }}{ }^{*}$, Milosevic, M. ${ }^{a}$, Antic, A. ${ }^{a}$, Borojevic, S. ${ }^{b}$, Ficko, M. ${ }^{c}$ \\ ${ }^{a}$ University of Novi Sad, Faculty of Technical Sciences, Novi Sad, Serbia \\ bUniversity of Banja Luka, Faculty of Mechanical Engineering, Banja Luka, Bosnia and Herzegovina \\ 'University of Maribor, Faculty of Mechanical Engineering, Maribor, Slovenia
}

\begin{abstract}
POVZETEK
Zaradi velikega števila tehničnih, tehnoloških, ekonomskih in okoljskih kriterijev je planiranje izdelovalnega postopka v fazi razvoja izdelka ena izmed težjih nalog. Izbor izdelovalnega postopka je kompleksen večkriterijski optimizacijski problem, ki mora upoštevati možne alternativne izdelovalne postopke in hkrati izpolniti številne zahteve izdelka. Članek predstavlja računalniško podprto metodologijo za večkriterijsko vrednotenje in izbor izdelovalnega postopka že v fazi konceptualnega planiranja izdelave. Razvita metodologija temelji na povezovanju oblike izdelka z izdelovalnimi zahtevami. Izločijo se izdelovalni postopki, ki ne izpolnjujejo desetih kriterijev, kot so material, obseg proizvodnje, produktivnost, dimenzijska natančnost, površinska obdelava itd. Nato se pripravi ocena in razvrstitev alternativ na osnovi petih kriterijev: časa izdelovalnega cikla, prilagodljivosti izdelovalnega postopka, izkoriščenosti materiala, kakovosti in stroškov izdelave. Pripravljen sistem za večkriterijsko izbiro izdelovalnega postopka je bil preizkušen na primeru izdelave endoproteze.
\end{abstract}

\section{PODATKI O ČLANKU}

Ključne besede: Izdelovalni postopki Konceptualno planiranje Večkriterijska izbira Izbira izdelovalnega postopka

*Kontaktna oseba: lukicd@uns.ac.rs (Lukic, D.)

Zgodovina članka:

Prejet 26. januarja 2017

Popravljen 24. februarja 2017

Sprejet 26. maja 2017 